\title{
SELEÇÃO DE ISOLADOS DE FUNGOS ENTOMOPATOGÊNICOS PARA O CONTROLE DE Hedypathes betulinus E AVALIAÇÃO DA PERSISTÊNCIA
}

\author{
Maria Sílvia Pereira Leite ${ }^{1}$, Edson Tadeu Iede ${ }^{2}$, Susete do Rocio Chiarello Penteado ${ }^{3}$, \\ Scheila Ribeiro Messa Zaleski ${ }^{4}$, Joelma Melissa Malherbe Camargo ${ }^{5}$, Rodrigo Daniel Ribeiro ${ }^{6}$ \\ ${ }^{1}$ Bióloga, Dra., Turfal Ind. Com. Prod. Biológicos e Agronômicos Ltda., Quatro Barras, PR, Brasil - silvia@turfal.agr.br \\ ${ }^{2}$ Biólogo, Dr., Embrapa Florestas, Colombo, PR, Brasil - iedeet@ cnpf.embrapa.br \\ ${ }^{3}$ Bióloga, Dra ., Embrapa Florestas, Colombo, PR, Brasil - susete@ cnpf.embrapa.br \\ ${ }^{4}$ Eng $^{\mathrm{a}}$ Agrônoma, Dra., Curitiba, PR, Brasil - srmzaleski@yahoo.com.br \\ ${ }^{5}$ Bióloga, M.Sc., Curitiba, PR, Brasil - melissajoelma@ yahoo.com.br \\ ${ }^{6}$ Biólogo, M.Sc., Curitiba, PR, Brasil - rdrcwb@yahoo.com.br
}

Recebido para publicação: 19/07/2010 - Aceito para publicação: 30/05/2011

\begin{abstract}
Resumo
Hedypathes betulinus (KLUG, 1825) (Coleoptera: Cerambycidae) é a principal praga da cultura da ervamate. Para o seu controle, avaliou-se em laboratório e campo a utilização de fungos entomopatogênicos. Em laboratório, foi avaliada a infectividade de isolados dos fungos Beauveria bassiana (Bals.) Vuillemin, 1912; B. brongniartii (Sacc.) Petch, 1926; Metarhizium anisopliae (Metsch.) Sorokin, 1883, e Paecilomyces (=Isaria) sp. Bainier, 1907, em adultos de H. betulinus. Os insetos foram imersos nas suspensões fúngicas na concentração de $10^{7}$ conídios $/ \mathrm{mL}$. Após a aplicação, eles foram mantidos em laboratório para registro da mortalidade. Verificou-se que B. bassiana (CG 716) foi o mais infectivo, com $100 \%$ de mortalidade. Foi estimada a $\mathrm{CL}_{50}$ do isolado $\mathrm{CG} 716$ de $B$. bassiana com cinco concentrações, variando de $10^{5}$ a $10^{9}$ conídios $/ \mathrm{mL}$, mais a testemunha. A $\mathrm{CL}_{50}$ estimada foi de $2,0 \times 10^{6}$ conídios $/ \mathrm{mL}$, variando de $6 \times 10^{5}$ a $5 \times 10^{6}$ conídios $/ \mathrm{mL}$. Foi avaliada a persistência do isolado CG 716 de B. bassiana formulado à base de óleo emulsionável em campo, verificando-se alta mortalidade nas primeiras três semanas após a aplicação do fungo, variando de 95 a 78\%, decaindo para 65 a 47\%, um e dois meses após a aplicação, respectivamente.

Palavras-chave: Controle biológico; broca-da-erva-mate; $\mathrm{CL}_{50}$.
\end{abstract}

\begin{abstract}
Entomopathogenic fungi in the control of Hedypathes betulinus and evaluation of persistence. Hedypathes betulinus (KLUG, 1825) (Coleoptera: Cerambycidae) is the main pest of "erva-mate" (Ilex paraguariensis) culture. Focusing its control, it was evaluated in laboratory and field the entomopathogenic fungi using. In laboratory, it was evaluated the infectiveness of the strains of the following fungi: Beauveria bassiana (Bals.) Vuillemin, B. brongniartii (Sacc.) Petch, Metarhizium anisopliae (Metsch.), Sorokin and Paecilomyces (= Isaria) sp. Bainier, in adults specimen of $H$. betulinus. The insects were immersed in a conidial suspension at a concentration of $10^{7}$ conidia $\mathrm{mL}^{-1}$. After the application the insects had been kept at laboratory for mortality register. It was verified that $B$. bassiana (CG 716) was more infective, reaching to $100 \%$ of mortality. It estimated $\mathrm{LC}_{50}$ of isolated CG 716 of $B$. bassiana with five concentrations and a variation of $10^{5}$ to $10^{9}$ conidia $\mathrm{mL}^{-1}$, along the control plot. The estimated $\mathrm{LC}_{50}$ was $2,0 \times 10^{6}$ conidia $\mathrm{mL}^{-1}$, ranging from $6 \times 10^{5}$ to $5 \times 10^{6}$ conidia $\mathrm{mL}^{-1}$. The persistence of strain CG 716 of B. bassiana - formulate oil was evaluated in field, producing the highest mortality, at the first three weeks after the application of the fungus, with a variance of 95 to $78 \%$, with a decreasing of 65 to $47 \%$, along one and two months after the application , respectively.

Keywords: Biological control; erva-mate pest; $\mathrm{LC}_{50}$.
\end{abstract}

\section{INTRODUÇÃO}

No Brasil, Hedypathes betulinus (Klug, 1825) (Coleoptera: Cerambycidae) é a principal praga da cultura erva-mate (Ilex paraguariensis St.-Hil.), distribuindo-se por toda a região produtora da erva-mate. 
Essa praga pertence ao grupo dos serradores (CÂNDIDO FILHO, 1929), sendo assim chamada por perfurar os galhos, troncos e raízes da planta. Os danos mais severos são ocasionados pelas larvas, as quais constroem galerias longitudinais ascendentes no tronco, que impedem a circulação normal da seiva e resultam no depauperamento das erveiras, podendo causar mortalidade das árvores (ALENCAR, 1960).

$\mathrm{O}$ uso de agrotóxicos, tanto para o controle de formas imaturas como de adultos, não é recomendável, tendo em vista a inexistência de princípios ativos que sejam comprovadamente eficientes e de baixa toxicidade para homens e animais, assim como a proibição do emprego desses produtos na ervamate, provavelmente devido ao risco de haver resíduos tóxicos no produto final (LEITE et al., 2006, apud BORGES, 2007).

Pagliosa et al. (1994) realizaram estudos para a implementação do controle microbiano de $H$. betulinus, avaliando em laboratório a infectividade de isolados do fungo Beauveria bassiana (Bals.) Vuillemin 1912, obtidos de bancos genéticos, isolados de outras espécies de insetos. Resultados promissores foram obtidos com o isolado CG 152, cuja infectividade foi de 73,4\%.

Soares et al. (1995) e Soares e Iede (1997), apud Borges (2007), detectaram a ocorrência natural dos fungos entomopatogênicos B. bassiana e Metarhizium anisopliae (Metsch.) Sorokin, 1883, em adultos de $H$. betulinus. Leite et al. (2000), apud Borges (2007), isolaram uma cepa de B. bassiana proveniente de adultos de $H$. betulinus coletados em campo, a qual foi incorporada e catalogada na Embrapa Recursos Genéticos e Biotecnologia, como CG 716. Esse isolado foi comparado a outros de $B$. bassiana, B. brongniartii (Sacc.) Petch, 1926, e M. anisopliae, apresentando-se como o mais eficiente, com alta infectividade em laboratório.

B. bassiana tem sido testada para o controle de diversos cerambicídeos, como em adultos de Plectrodera scalator Fabricius, 1792, praga de Populus deltoides Marsh (cottonwood), em Kentucky, Estados Unidos, atingindo porcentagens de mortalidade superiores a 60\% (FORSCHLER; NORDIN, 1988). Na China, B. bassiana tem sido produzida em larga escala e utilizada no controle de mais de 30 espécies de pragas florestais e agrícolas (FENG et al., 1994).

Como esses patógenos são muito sensíveis às condições do ambiente, para obterem maior estabilidade quando utilizados em campo é necessário que eles sejam formulados. Formulações à base de óleo, além de aumentar a sobrevivência dos conídios, auxiliam sua adesão ao tegumento do inseto, protegendo-os contra a dissecação e os raios ultravioleta, facilitando também o manuseio para o aplicador. O óleo auxilia na adesão dos conídios nas superfícies hidrofóbicas da cutícula dos insetos e das plantas (PRIOR et al., 1988; JOHNSON; GOETTEL, 1993).

Depois da aplicação do patógeno no meio ambiente, o seu destino varia de acordo com a espécie do fungo e com os fatores ambientais. A sua persistência, o seu crescimento populacional e a sua dispersão são os principais fatores que afetam seu destino. Como os vírus e as bactérias, os fungos entomopatogênicos apresentam uma grande persistência no solo e na população hospedeira (FUXA, 1992). B. bassiana pode persistir no solo por períodos de 15 dias a 1 ano, após a liberação (WATT; LEBRUN, 1984; MÜLLER-KÖGLER; ZIMMERMAN, 1986). Thorvilson et al. (1985) e Daoust e Pereira (1986) observaram que B. bassiana tem persistência de 32 semanas em cadáveres hospedeiros.

Os objetivos do presente estudo foram realizar a seleção de isolados de fungos entomopatogênicos visando o controle de $H$. betulinus e avaliar a persistência em campo. Espera-se que, com este estudo, possa ser encontrado um agente controlador da praga em pelo menos $70 \%$ de sua população e que possua persistência acima de um mês.

\section{MATERIAL E MÉTODOS}

Os experimentos de laboratório e de campo foram conduzidos na Embrapa Florestas Colombo, PR.

Os insetos utilizados nos bioensaios laboratoriais foram coletados em ervais localizados nos municípios de Ivaí (PR) e São Bento do Sul (SC). Em função da dificuldade de os insetos serem criados em laboratório, e para garantir a obtenção de insetos sadios e com poucos dias de vida, foram selecionados apenas os que apresentavam o corpo todo recoberto por pêlos de coloração branca (característica de insetos recém-emergidos) e sem danos, como ruptura de antenas e pernas. Em laboratório, os insetos foram selecionados e individualizados em copos plásticos transparentes, medindo $11 \mathrm{~cm}$ de altura x $8 \mathrm{~cm}$ de diâmetro, sendo ofertados ramos de erva-mate para sua alimentação. 
Seleção dos isolados

Para avaliar a infectividade de diferentes isolados de fungos entomopatogênicos em adultos de H. betulinus, foi realizado um bioensaio com os seguintes fungos: Beauveria bassiana (CG 152, 61, 15, 25, 544, 21, 716 e BB 01); B. brongniartii (CG 72); Metarhizium anisopliae (CG 100) e Paecilomyces (= Isaria) sp. (CG 815), conforme tabela 1. Os isolados foram cultivados em meio de arroz e incubados a $24 \pm 2{ }^{\circ} \mathrm{C}$ durante 20 dias. Para avaliar a viabilidade dos conídios dos isolados, eles foram plaqueados em meio de ágar-água. Após 24 h foi quantificada, em microscópio óptico, a porcentagem de germinação de 100 conídios, considerando-se conídio germinado aquele que apresentava o tubo germinativo. Posteriormente, os conídios dos isolados foram quantificados em câmara de Neubauer e ajustados na concentração de $3,5 \times 10^{7}$ conídios/mL. Adultos de H. betulinus foram imersos por um segundo nas suspensões compostas por conídios do fungo, água estéril e Tween 70\%. A mesma metodologia foi adotada para a testemunha, porém sem o fungo. Após a imersão, os insetos foram individualizados em copos plásticos, contendo em sua base papel filtro. Ramos de erva-mate foram ofertados como alimento e as observações foram diárias, até a morte dos insetos. Após a morte, procedeu-se à imersão dos insetos em álcool $70 \%$ e água destilada e posterior individualização em câmara úmida, para se observar a extrusão do fungo e a confirmação do agente causal. O experimento constou de um delineamento experimental inteiramente casualizado, com 11 tratamentos e 30 repetições, sendo cada repetição constituída de um inseto adulto. O experimento foi conduzido em salas climatizadas com temperatura de $24 \pm 2{ }^{\circ} \mathrm{C}$, UR de $70 \pm 10 \%$ e fotofase de 12 horas. A eficiência dos isolados foi avaliada mediante a percentagem de mortalidade. Os dados referentes à mortalidade foram submetidos à análise de variância $\mathrm{e}$ as médias comparadas pelo teste de Tukey $(\mathrm{P} \leq 0,05)$.

Tabela 1. Origem e hospedeiros dos isolados dos fungos entomopatogênicos utilizados nos testes de laboratório e campo.

Table 1. Origin and host of the isolates of the entomopathogenic fungi used in laboratory tests and field.

\begin{tabular}{|c|c|c|}
\hline Isolados & Hospedeiros & Origem \\
\hline & \multicolumn{2}{|c|}{ 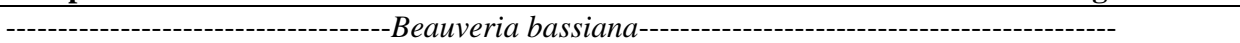 } \\
\hline CG 716 & Hedypathes betulinus (Coleoptera: Cerambycidae) & Colombo - PR \\
\hline CG 152 & (Coleoptera: Chrysomelidae) & Goiânia - GO \\
\hline CG 61 & Diabrotica sp. (Coleoptera: Chrysomelidae) & Brasília - DF \\
\hline CG 15 & Lebia concinna (Coleoptera: Carabidae) & Londrina - PR \\
\hline CG 25 & Anticarsia gemmatalis (Lepidoptera: Noctuidae) & Brasília - DF \\
\hline CG 544 & Rhinostomus barbirostris (Coleoptera: Curculionidae) & Cenargen \\
\hline BB 01 & Hedypathes betulinus (Coleoptera: Cerambycidae) & São Bento do Sul - SC \\
\hline & ----------------------------------Beauveria brongniartii-- & -------------- \\
\hline CG 72 & Diatraea saccharalis (Lepidoptera: Pyralidae) & Araras - SP \\
\hline CG 100 & (Coleoptera: Cerambycidae) & Ivaí - PR \\
\hline CG 815 & $\begin{array}{l}\text { Hedypathes betulinus (Coleoptera: Cerambycidae) } \\
\text { ( }\end{array}$ & Ivaí - PR \\
\hline
\end{tabular}

Concentração média letal

Para a estimativa da concentração média letal $\left(\mathrm{CL}_{50}\right)$ de conídios, foi selecionado o isolado CG 716 de B. bassiana, em função de ter apresentado a maior porcentagem de mortalidade (100\%) e de ter sido, entre os fungos isolados do próprio inseto, o isolado que apresentou o menor $\mathrm{TL}_{50}$ ( 22,9 dias). $\mathrm{O}$ delineamento experimental foi inteiramente casualizado, com seis tratamentos (cinco concentrações de conídios do fungo e a testemunha) e 20 repetições. O fungo foi multiplicado em meio de arroz, conforme descrito no experimento anterior. Os conídios foram quantificados nas concentrações de $5 \times 10^{5}, 3 \times 10^{6}$, $5 \times 10^{7}, 3 \times 10^{8}$ e $4 \times 10^{9}$ conídios $/ \mathrm{mL}$, e adultos de $H$. betulinus foram imersos por um segundo nas suspensões compostas por conídios de B. bassiana, água estéril e Tween $70 \%$. A mesma metodologia foi adotada para a testemunha, porém sem o fungo. Posteriormente, os insetos foram individualizados em potes plásticos, sendo ofertados ramos de erva-mate até a morte dos insetos. Os procedimentos para a constatação da extrusão do fungo nos insetos mortos foram iguais aos do experimento anterior. $\mathrm{O}$ experimento foi conduzido em sala climatizada, com temperatura de $22 \pm 2{ }^{\circ} \mathrm{C}$, UR de $70 \pm 10 \%$ e fotofase 
de 12 horas. A estimativa da $\mathrm{CL}_{50}$ foi efetuada mediante a utilização do programa computacional Micro Probit (versão 3.0). Os dados referentes à mortalidade foram submetidos à análise de variância e as médias comparadas pelo teste de Tukey $(\mathrm{P} \leq 0,05)$.

Persistência

Para avaliar a persistência do isolado CG 716 de $B$. bassiana formulado à base de óleo vegetal emulsionável em campo, instalou-se um experimento, em blocos ao acaso, em um erval localizado na Embrapa Florestas, em Colombo (PR), com aproximadamente 10 anos de idade e espaçamento de $3 \times 2 \mathrm{~m}$. Foram selecionadas, ao acaso, cinco erveiras, as quais foram podadas previamente e cada uma acondicionada em uma gaiola confeccionada em madeira e tela metálica, medindo $1,50 \times 1,60 \mathrm{~m}$. A distância entre as gaiolas era de aproximadamente 10 metros, com exceção da testemunha, que equidistou 100 metros das demais. O fungo foi aplicado em quatro erveiras, na concentração de 4,6x10 $0^{8}$ conídios $/ \mathrm{mL}$ mais $1 \%$ de óleo emulsionável. Utilizou-se uma erveira como testemunha, adotando-se a mesma metodologia de aplicação, porém sem o fungo. A suspensão foi aplicada com pulverizador costal, durante 15 segundos, apenas no tronco da erveira, utilizando-se $150 \mathrm{~mL} /$ planta, sendo, posteriormente, colocados cinco insetos adultos na base do tronco de cada erveira. O experimento constou de nove tratamentos e quatro repetições. Os tratamentos foram: T1: insetos colocados logo após a aplicação do fungo; T2: insetos colocados $48 \mathrm{~h}$ após a aplicação do fungo; T3: insetos colocados $72 \mathrm{~h}$ após a aplicação do fungo; T4: insetos colocados 1 semana após a aplicação do fungo; T5: insetos colocados 2 semanas após a aplicação do fungo; T6: insetos colocados 3 semanas após a aplicação do fungo; T7: insetos colocados 1 mês após a aplicação do fungo; T8: insetos colocados 2 meses após a aplicação do fungo; e T9: testemunha, sem aplicação do fungo. Esse experimento foi iniciado no mês de dezembro e finalizado no início de fevereiro, com temperatura média de $21 \pm 2{ }^{\circ} \mathrm{C}$ e UR de $79 \pm 10 \%$. Foram utilizados 200 adultos de $H$. betulinus, na proporção de 4 machos para 1 fêmea, com 40 repetições por tratamento. Em todos os tratamentos os insetos permaneceram $24 \mathrm{~h}$ nas gaiolas e posteriormente foram recoletados, identificados e individualizados em copos plásticos com alimento, permanecendo em laboratório até a sua morte, em condições não controladas, com temperatura média de $20 \pm 2{ }^{\circ} \mathrm{C}$ e UR de $66,9 \pm 10 \%$. Os procedimentos para verificar a extrusão do fungo nos insetos mortos foram iguais aos dos experimentos anteriores. Os dados referentes à mortalidade foram analisados através de análise de regressão.

\section{RESULTADOS E DISCUSSÃO}

\section{Seleção dos isolados}

Os sintomas de infecção foram observados inicialmente por tremores nas pernas, diminuição da alimentação, ocorrendo a extrusão do fungo, primeiramente, nas inserções abdominais do corpo, aparelho bucal, antenas e pernas, produzindo conídios de coloração branca. Segundo Alves (1998), o inseto infectado cessa a alimentação, tornando-se fraco e visivelmente desorientado, aparecendo um halo branco associado à estrutura característica do conidióforo e dos conídios do fungo.

A germinação dos conídios de todos os isolados foi superior a $90 \%$. Os valores referentes às percentagens de mortalidade de adultos de $H$. betulinus e $\mathrm{TL}_{50}$, causados por diferentes isolados dos fungos avaliados, encontram-se na tabela 2. Os isolados que apresentaram alta infectividade foram: CG 716 e BB 01 do fungo B. bassiana, os quais não diferiram estatisticamente entre si, com infectividade de $95,8 \%$ e $100 \%$, respectivamente. Os isolados que apresentaram infectividade moderada foram B. bassiana CG 544, CG 15, CG 61, CG 152 e Paecilomyces (= Isaria) sp. CG 815, com mortalidade de 37,5\% a 83\%, diferindo estatisticamente entre si. Os isolados CG 100 (M. anisopliae), CG 72 (B. brongniartii) e CG 25 (B. bassiana) não diferiram estatisticamente da testemunha, mostrando baixa infectividade, variando de $2,1 \%$ a $31,2 \%$. Na testemunha não foi verificada a ocorrência de fungos entomopatogênicos em nenhuma das repetições, entretanto a mortalidade foi de $10,4 \%$, por causas naturais. Pagliosa et al. (1994) encontraram para o isolado CG 152, de B. bassiana, uma infectividade de 73,4\% em adultos de $H$. betulinus, em laboratório.

A patogenicidade diferenciada entre os isolados dos fungos avaliados indica que os isolados CG 716 e BB 01 de B. bassiana, coletados em adultos de H. betulinus, apresentam maior virulência a essa 
praga. De acordo com Alves (1998), quando as doenças de insetos ocorrem naturalmente nas populações de pragas, devem-se considerar esses patógenos como elementos básicos no manejo integrado da praga, dependendo principalmente do uso de medidas que favoreçam o bom desenvolvimento da epizootia. Shimazu et al. (2002) avaliaram a patogenicidade de isolados dos fungos B. bassiana, M. anisopliae e Paecilomyces (= Isaria) sp. em larvas do cerambicídeo Anoplophora glabripennis (Motschulsky, 1853). $\mathrm{O}$ fungo foi aplicado nas larvas em uma concentração de conídios semelhante à deste experimento, 1,0x10 conídios/mL, sendo o fungo B. bassiana F0003 o mais infectivo, causando $100 \%$ de mortalidade. Os resultados demonstram que para essas duas espécies de cerambicídeos o fungo $B$. bassiana foi o que causou os maiores índices de mortalidade.

O cálculo do $\mathrm{TL}_{50}$ indicou uma variação entre os isolados de 9,8 a 32 dias, sendo que os isolados CG 61, CG 15, CG 152 e CG 72 apresentaram os menores valores, com 9; 8; 10;10,1 e 14,3 dias, respectivamente (Tabela 2). Os isolados obtidos de $H$. betulinus apresentaram $\mathrm{TL}_{50}$ superiores aos isolados obtidos de outros insetos. Isso também pode ser comprovado quando se compara o isolado mais infectivo obtido de H. betulinus (CG 716), que apresentou um $\mathrm{TL}_{50}$ de 22,9 dias, com o CG 152 testado por Pagliosa et al. (1994), que foi obtido de outra espécie de inseto e apresentou um $\mathrm{TL}_{50}$ de 9,4 dias. Shimazu et al. (2002) obtiveram um $\mathrm{TL}_{50}$ de 16 dias para Anoplophora glabripennis, período também inferior ao encontrado para $H$. betulinus. O tempo médio para causar a mortalidade pode estar atribuído a vários fatores, entre eles a infectividade do isolado e a resistência do inseto. Para a seleção do isolado mais virulento para testes em campo, foram avaliados a infectividade, o $\mathrm{TL}_{50}$ e a origem dos isolados. Optou-se pelo isolado CG 716 por ter sido um dos mais virulentos e por ter sido obtido de H. betulinus, pois, para utilização em campo, é necessário levar em consideração isolados que já estejam adaptados às condições climáticas do ambiente de origem, para causar epizootias.

Tabela 2. Eficiência de isolados de B. bassiana, B. brongniartii, M. anisopliae e Paecilomyces (= Isaria) sp. no controle de $H$. betulinus, em laboratório $(\mathrm{n}=30)$.

Table 2. Efficiency of isolates of B. bassiana, B. brongniartii, M. anisopliae and Paecilomyces (= Isaria) $\mathrm{sp}$. in the control of H. betulinus in the laboratory $(\mathrm{n}=30)$.

\begin{tabular}{lcc}
\hline Isolados & Mortalidade (\%) & TL $_{\mathbf{5 0}}$ (dias) $\mathbf{I C}^{(\mathbf{( 1 )}}$ \\
\hline B. bassiana CG 716 & $100 \pm 0,00 \mathrm{a}$ & $22,9 \pm 2,09 \mathrm{a}(18,56-27,19)$ \\
B. bassiana BB 01 & $95,8 \pm 4,08 \mathrm{a}$ & $26,4 \pm 2,47 \mathrm{a}(21,28-31,49)$ \\
B. bassiana CG 544 & $90,0 \pm 6,71 \mathrm{ab}$ & $18,5 \pm 2,24 \mathrm{a}(21,38-30,71)$ \\
B. bassiana CG 15 & $83,0 \pm 5,48 \mathrm{ab}$ & $10,0 \pm 0,40 \mathrm{~b}(9,30-10,91)$ \\
B. bassiana CG 61 & $76,6 \pm 6,04 \mathrm{ab}$ & $9,8 \pm 0,48 \mathrm{~b}(8,83-10,79)$ \\
B. bassiana CG 152 & $66,6 \pm 6,80 \mathrm{~b}$ & $10,1 \pm 0,51 \mathrm{~b}(9,46-11,54)$ \\
Paecilomyces (= Isaria) sp. CG 815 & $37,5 \pm 7,65 \mathrm{c}$ & $32,0 \pm 1,38 \mathrm{a}(29,19-34,80)$ \\
M. anisopliae CG 100 & $31,2 \pm 11,59 \mathrm{~cd}$ & $25,8 \pm 2,70 \mathrm{a}(20,07-31,53)$ \\
B. brongniartii CG 72 & $31,1 \pm 6,90 \mathrm{~cd}$ & $14,3 \pm 1,15 \mathrm{~b}(11,45-16,12)$ \\
B. bassiana CG 25 & $2,1 \pm 2,04 \mathrm{~d}$ & $17,0 \pm 0,00 \mathrm{ab}$ \\
Testemunha & $10,45 \pm 3,74 \mathrm{~d}$ & $27,7 \pm 2,21 \mathrm{a}(3,29-12,13)$ \\
\hline
\end{tabular}

${ }^{(1)}$ : Médias seguidas pelas mesmas letras na coluna não diferem estatisticamente pelo teste de Tukey a $5 \%$ de probabilidade. ${ }^{(2)}$ : Intervalo de confiança.

Concentração média letal. A $\mathrm{CL}_{50}$ estimada para o isolado CG 716 é de $2 \times 10^{6}$ conídios $/ \mathrm{mL}$, variando de $6 \times 10^{5}$ a $5 \times 10^{6}$ conídios $/ \mathrm{mL}$ (Tabela 3 ).

As concentrações de $10^{7}, 10^{8}$ e $10^{9}$ conídios/mL causaram as mais elevadas porcentagens de mortalidade (84, 95 e $100 \%$, respectivamente), não diferindo estatisticamente entre si. Shimazu et al. (2002) estimaram a $\mathrm{CL}_{50}$ para os isolados F-263 (B. bassiana) sobre larvas e adultos de A. glabripennis. A metodologia de aplicação foi semelhante à utilizada para $H$. betulinus, porém as concentrações utilizadas foram inferiores, $10^{3}, 10^{4}, 10^{5}, 10^{6}$ e $10^{7}$ conídios $/ \mathrm{mL}$, apresentando $\mathrm{CL}_{50}$ semelhante à deste bioensaio, $4,1 \times 10^{6}$ conídios $/ \mathrm{mL}$, variando de $3,4 \times 10^{5}$ a $5,0 \times 10^{7}$ conídios $/ \mathrm{mL}$. Em relação ao $\mathrm{TL}_{50}$, a concentração de $10^{9}$ conídios $/ \mathrm{mL}$ diferiu significativamente das outras concentrações avaliadas, apresentando 13,2 dias. Já para $10^{8}, 10^{7}, 10^{6}$ e $10^{5}$ conídios $/ \mathrm{mL}$, foi encontrado um $\mathrm{TL}_{50}$ de 20,2; 20,7; 27,6 e 23,4 dias, respectivamente. Shimazu et al. (2002) obtiveram um $\mathrm{TL}_{50}$ inferior para F-263, de 8,1 a 10 dias. 
Tabela 3. Mortalidade média ( $\pm \mathrm{EP}$ ) de adultos de $H$. betulinus ocasionada pelo isolado CG 716 de $B$. bassiana, em diferentes concentrações, em laboratório $(\mathrm{n}=20)$.

Table 3. Mortality Mean ( \pm SE) of adult $H$. betulinus caused by isolate CG 716 B. bassiana at different concentrations, in the laboratory $(\mathrm{n}=20)$.

\begin{tabular}{|c|c|c|c|}
\hline $\begin{array}{l}\text { Concentração } \\
\text { (conídios/mL) }\end{array}$ & $\begin{array}{c}\text { Mortalidade } \\
(\%)^{(1)}\end{array}$ & $\begin{array}{l}\text { TL }_{50}(\text { dias }) \\
\text { IC }^{(2)}\end{array}$ & $\begin{array}{c}\mathrm{CL}_{50}(\text { conídios/mL) } \\
\text { IC }^{(2)}\end{array}$ \\
\hline 0 & $0,0 \pm 0,00 \mathrm{c}$ & $0,0 \pm 0,00 \mathrm{~b}$ & $2,0 \times 10^{6}$ \\
\hline $5,5 \times 10^{5}$ & $42,1 \pm 11,33 b$ & $23,4 \pm 2,05$ a $(19,09-27,66)$ & $\left(6 \times 10^{5}-5 \times 10^{6}\right)$ \\
\hline $3,3 \times 10^{6}$ & $39,0 \pm 11,49 b$ & $27,6 \pm 1,61$ a $(24,18-30,96)$ & - \\
\hline $4,9 \times 10^{7}$ & $84,2 \pm 8,37 \mathrm{a}$ & $20,7 \pm 1,49$ a $(17,56-23,81)$ & - \\
\hline $3,2 \times 10^{8}$ & $95,0 \pm 4,87 \mathrm{a}$ & $20,2 \pm 1,2929$ a $(17,51-22,91)$ & - \\
\hline $3,8 \times 10^{9}$ & $100,0 \pm 0,00 \mathrm{a}$ & $13,2 \pm 1,53 \mathrm{~b}(10,01-16,40)$ & - \\
\hline
\end{tabular}

Persistência

Verifica-se que o formulado fúngico utilizado neste experimento foi infectivo durante todo o período de avaliação (60 dias) (Figura 1). Nas primeiras três semanas após a aplicação, o fungo causou alta mortalidade, variando de 95 a 78\%, decaindo para 65 a 47\%, um e dois meses após a aplicação, respectivamente. O tempo médio de mortalidade variou de 20 a 35 dias. A testemunha apresentou mortalidade por causas naturais de 3\% nas primeiras três semanas e de $7 \%$ um e dois meses após a aplicação, não apresentando nenhuma infecção por B. bassiana. Segundo Engler e Rogoff (1976), a rápida inativação dos entomopatógenos em condições de campo é devida às elevadas temperaturas, esterilização pela irradiação solar e microambientes na superfície das folhas, incluindo $\mathrm{pH}$ e os desconhecidos inativadores liberados pelas plantas. Conídios de B. bassiana são altamente suscetíveis ao detrimental efeito da luz solar (FARGUES et al., 1996). A luz solar na faixa de 290 a $400 \mathrm{~nm}$ pode afetar a persistência dos fungos depositados nas folhagens (FUXA, 1987). Ambientes com temperaturas acima de $35^{\circ} \mathrm{C}$ reduzem a persistência, apresentando as temperaturas baixas menores efeitos (McCOY et al., 2006).

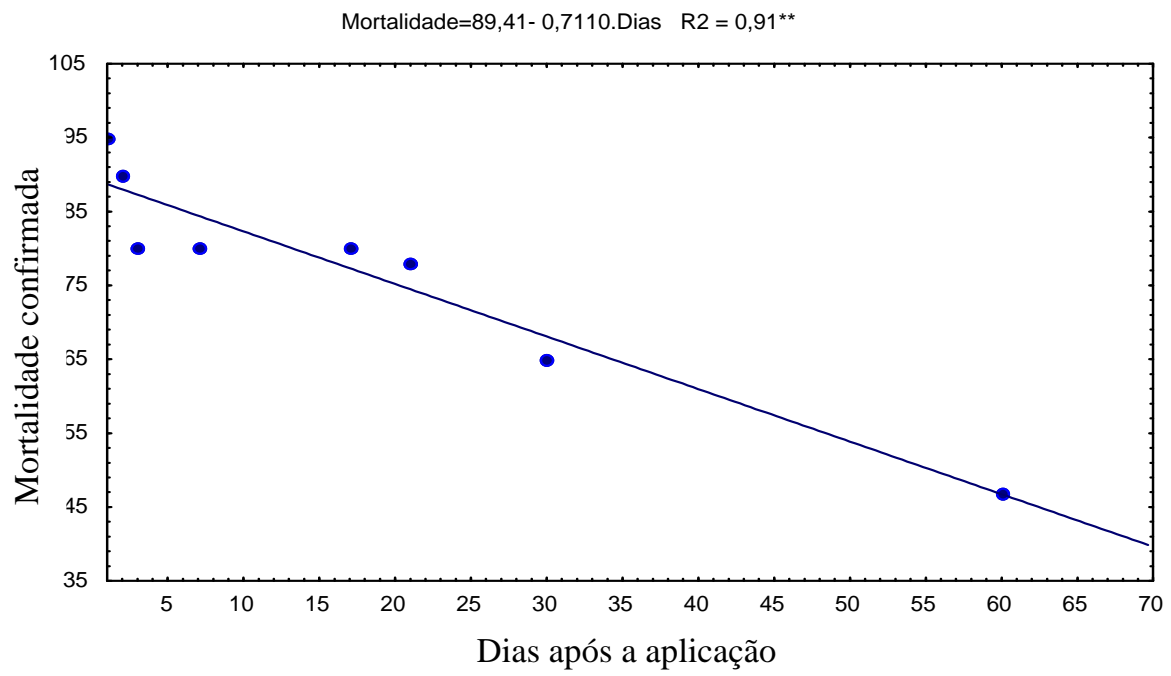

Figura 1. Persistência de B. bassiana avaliada em adultos de H. betulinus.

Figure 1. Persistence of B. bassiana evaluated in adults of $H$. betulinus.

Durante a avaliação do experimento, vários fatores auxiliaram na persistência do fungo, como as condições climáticas favoráveis, com U.R. média de $79,1 \pm 10 \%$ e temperatura média de $21 \pm 2{ }^{\circ} \mathrm{C}$, a 
aplicação do formulado no tronco, uma região da erveira que fica protegida da insolação direta, e a formulação em óleo, que auxiliou na proteção dos conídios.

Como os adultos de $H$. betulinus têm o hábito de caminhar e ovipositar no tronco da erveira, a aplicação do fungo direcionada é estratégica para atingir o inseto. Esse tipo de aplicação também limita a infecção de outros insetos associados às folhas e galhos da erva-mate, principalmente os inimigos naturais, além de não deixar resíduos nas folhas. O objetivo do controle microbiano é o controle da praga a um nível populacional que não chegue a ponto de ser prejudicial, pois a presença de um número mínimo desses insetos é favorável para a manutenção da população de predadores, parasitoides e patógenos que estão presentes no agroecossistema (ALVES, 1998).

De acordo com Luz et al. (1999), o desenvolvimento das formulações à base de óleo dos fungos entomopatogênicos é uma estratégia para preservá-los dos efeitos ambientais negativos. As formulações de fungos entomopatogênicos à base de óleo são superiores àquelas à base de água, especialmente em locais onde a umidade é baixa (BATEMAN et al., 1993). Formulações em óleo prolongam a sobrevivência do conídio e diminuem a sensibilidade a radiações UV quando comparada com suspensões aquosas (INGLIS et al., 1995).

A eficiência de fungo entomopatogênico na produção de uma cultura irá depender da persistência do inóculo em campo. Embora existam exceções, a persistência dos fungos entomopatogênicos em diferentes partes da planta é de poucos dias. B. bassiana permanece viável de 5 a 21 dias na superfície da planta (GARDNER et al., 1977; IGNOFFO et al., 1979). Inglis et al. (1995) reportaram que a chuva pode afetar a persistência de B. bassiana na folhagem. Os conídios de B. bassiana são mais persistentes quando depositados no solo (VESTERGAARD et al., 2003). Inglis et al. (1997) relataram que, depois de um inicial declínio nos primeiros 20 dias após a aplicação dos conídios, a persistência se estabiliza por aproximadamente 200 dias. O fungo $B$. bassiana foi mais eficiente no controle de $H$. betulinus quando comparado com os fungos $B$. brongniartii, M. anisopliae e Paecilomyces (= Isaria) sp., sendo o isolado CG 716 um dos mais infectivos, selecionado para estudos de persistência a campo. Esse isolado formulado em óleo emulsionável apresentou persistência durante os 60 dias de avaliação.

\section{CONCLUSÕES}

O fungo, B. bassiana CG 716 demonstrou ser um agente promissor para o manejo integrado da broca-da-erva-mate, apresentando controle acima de $70 \%$ da população da praga $(95,8 \%)$, com persistência acima de mês.

\section{AGRADECIMENTOS}

Ao Sr. Paulo Corrêa, pela concessão da área experimental na região de Campo Alegre, SC.

À ervateira Bitumirim, pelo incentivo à pesquisa e fornecimento de insetos para os bioensaios.

\section{REFERÊNCIAS}

ALENCAR, F. R. de. Erva-mate. Serviço de Informação Agrícola, Rio de Janeiro, p. 10 - 23, 1960.

ALVES, S. B. Microorganismos associados a insetos. In: ALVES, S. B. Controle microbiano de insetos. Piracicaba: FEALQ, 1998. p. 75 - 96.

BATEMAN, R. P.; CAREY, M.; MOORE, D.; PRIOR, C. The enhanced infectivity of Metarhizium flavoviride in oil formulations to desert locust at low humites. Annals of Applied Biology, v. 122, p. 145 - 152. 1993.

BORGES, L. R. Eficiência de Beauveria bassiana (Bals.) Vuill. (Deuteromycota) para o controle de Hedypathes betulinus (Klug) (Coleoptera: Cerambycidae) em erva-mate, Ilex paraguariensis St.-Hil. (Aquifoliaceae). 102 p. Tese (Doutorado em Entomologia) - Universidade Federal do Paraná, Curitiba, 2007. 
CÂNDIDO FILHO, J. A. A broca da herva-mate (Hedypathes betulinus Klug). In: O Mate. Curitiba, 1929. v. 1. p.13 - 14 .

DAOUST, R. A.; PEREIRA, R. M. Survival of Beauveria bassiana (Deuteromycetes: Moniliales) conidia on cadavers of cowpea pests stored outdoors and in laboratory in Brazil. Environmental Entomology, v. 15 , p. $642-647,1986$.

ENGLER, R.; ROGOFF, M. H. Entomopathogens: ecological manipulation of natural associations. Environmental Health Perspectives, v. 14, p. 153 - 159, 1976.

FARGUES, J.; GOETTEL, M. S.; SMITS, N.; OUEDRAOGO, A.; VIDAL, C.; LACEY, L. A.; LOMER, C. J.; ROUGIER, M. Variability in susceptibility to simulated sunlight of conidia among isolates of entomopathogenic Hyphomycetes. Mycopathologia. v. 135, p. 171 - 181, 1996.

FENG, M. G.; POPRAWSKI, T. J.; KHACHATOURIANS, G. G. Production, formulation and application of the entomopathogenic fungus Beauveria basssiana for insect control: current status. Biocontrol Science and Technology, v. 4, p. 3 - 34, 1994.

FORSCHLER, B. T.; NORDIN, G. L. Comparative pathogenicity of selected entomogenous nematodes to the hardwood borers, Prionoxystus robiniae (Lepidoptera: Cossidae) and Megacyllene robiniae (Coleoptera: Cerambycidae). Journal of Invertebrate Pathology, v. 52, n. 2, p. 343 - 347, 1988.

FUXA, J. R. Ecological considerations for the use of entomopathogens in IPM. Annual Reviews Entomology, v. 32, p. 225 - 251, 1987.

Impact of the release of entomopathogens in the environment. Pesquisa Agropecuária Brasileira, v. 27, 1992.

GARDNER, W. A.; SUTTON, R. M.; NOBLET, R. Persistence of Beauveria bassiana, Nomuraea rileyi, and Nosema necatrix on soybean foliage. Environmental Entomology, v. 6, p. 616 - 618, 1977.

IGNOFFO, C. M.; GARCIA, C.; ALYOSHINA, O. A.; LAPPA, N. V. Laboratory and field studies with Boverin: a mycoinsecticidal preparation of Beauveria bassiana produced in the Soviet Union. Journal of Economic Entomology, v.72, p. 562 - 565, 1979.

INGLIS, G. D.; JOHNSON, D. L.; GOETTAL, M. S. Effects of simulated rain on the persistence of Beauveria bassiana on leaves of alfafa and wheat. Biocontrol Science and Technology, v. 5, p. 365 $369,1995$.

INGLiS, G. D.; DUKE, G. M.; KANAGARATNAM, P.; JOHNSON, D. L.; GOETTAL, M. S. Persistence of Beauveria bassiana in soil following application of conidia through crop canopies. Memory Entomological Society of Canada, v. 171, p. 253 - 263, 1997.

JOHNSON, D. L.; GOETTEL. M. S. Reduction of grasshopper populations following field application of the fungus Beauveria bassiana. Biocontrol Science and Technology, v. 3, p. 165 - 175, 1993.

LUZ, C.; SILVA, I. G.; MAGAlHÃES, B. P.; CORDEIRO, C. M. T.; TIGANO, M. S. Control of Triatoma infestans (Reduviidae, Tria-tominae) with Beauveria bassiana: preliminary assays on formulation and application in the field. Sociedade Entomológica Brasileira, n. 28. p. 101 - 110, 1999.

McCOY, C.; QUINTELA, E. D.; FARIA, M. Environmental persistence of entomopathogenic fungi. Disponível em: <www.isuagcenter.com/s265/mccoy.htm>. Acesso em: 12/09/2006.

MÜLLER-KÖGLER, E.; ZIMMERMAN, G. On the viability of Beauveria bassiana (Bals.) Vuill. In contaminated soil under field and laboratory conditions. Entomophaga, v. 31, p. 285 - 292, 1986.

PAGLIOSA, M. M. R.; SANTOS, H. R. dos; DIODATO, M. A. Patogenicidade do fungo entomopatogênico Beauveria bassiana (Bals.) Vuill., em Hedypathes betulinus (Klug, 1825), praga da erva-mate, Ilex paraguariensis St.-Hil. Agrárias, Curitiba, n. 13, v. 1/2, p. 229 - 231, 1994. 
PRIOR, C.; JOLLANDS, P.; LE PATOUREL, G. Infectivity of oil and water formulations of Beauveria bassiana (Deuteromycotina: Hyphomycetes) to the cocoa weevil pest Pantorhytes plutus (Coleoptera: Curculionidae). Journal of Invertebrate Pathology, v. 52, p. 66 - 72, 1988.

SHIMAZU, M.; ZHANG, B.; LIU, Y. Fungal pathogens of Anoplophora glabripennis (Coleoptera: Cerambycidade) and their virulences. Bulletin of FFPRI. L1, p. 1123 - 1130, 2002.

THORVILSON, H. G.; LEWIS, L. C.; PEDIGO, L. P. Overwintering potential of Nomuraea riley (Fungi: Deuteromycotina) from Plathypena scabra (Lepidoptera: Noctuidae) cadavers in central Iowa. Journal Kansas Entomology Society, v. 58, p. 662 - 667, 1985.

VESTERGAARD, S.; CHERRY, A.; KELLER, S.; GOETTEL, M. Safety of Hyphomycete fungi as microbial control agents. In: Environmental Impacts of Microbial Insecticides. Ed. Hokkanen, H. M. T. University of Helsinki, Finland. p. 35 - 62. 2003.

WATT, B. A.; LEBRUN, R. A. Soil effects of Beauveria bassiana on pupal populations of the Colorado potato beetle (Coleoptera: Chrysomelidae). Environmental Entomology, v. 13, p. 15 - 18, 1984. 
FLORESTA, Curitiba, PR, v. 41, n. 3, p. 619-628, jul./set. 2011.

Leite, M. S. P. et al. 